\title{
Real-Time Procurement Strategies of a Proactive Distribution Company with Aggregator-Based Demand Response
}

Zhang, Chunyu; Wang, Qi; Wang, Jianhui; Pinson, Pierre; Morales González, Juan Miguel; Østergaard, Jacob

Published in:

IEEE Transactions on Smart Grid

Link to article, DOI:

10.1109/TSG.2016.2565383

Publication date:

2016

Document Version

Peer reviewed version

Link back to DTU Orbit

Citation (APA):

Zhang, C., Wang, Q., Wang, J., Pinson, P., Morales González, J. M., \& Østergaard, J. (2016). Real-Time

Procurement Strategies of a Proactive Distribution Company with Aggregator-Based Demand Response. IEEE Transactions on Smart Grid, 9(2), 766 - 776. https://doi.org/10.1109/TSG.2016.2565383

\section{General rights}

Copyright and moral rights for the publications made accessible in the public portal are retained by the authors and/or other copyright owners and it is a condition of accessing publications that users recognise and abide by the legal requirements associated with these rights.

- Users may download and print one copy of any publication from the public portal for the purpose of private study or research.

- You may not further distribute the material or use it for any profit-making activity or commercial gain

- You may freely distribute the URL identifying the publication in the public portal 


\title{
Real-Time Procurement Strategies of a Proactive Distribution Company with Aggregator-Based Demand Response
}

\author{
Chunyu Zhang, Member, IEEE, Qi Wang, Member, IEEE, Jianhui Wang, Senior Member, IEEE, \\ Pierre Pinson, Senior Member, IEEE, Juan M. Morales, Member, IEEE, and Jacob Østergaard, Senior \\ Member, IEEE
}

\begin{abstract}
In this paper, we present a real-time trading framework for distribution networks where a rational aggregator is identified as a broker by contracting with individual demands and dealing with the distribution company. Demand response capability is characterized by the coexistence of elastic and inelastic demand components. A one-leader multi-follower bilevel model is proposed to derive the procurement strategies, i.e., the upper-level problem intends to maximize the profit of the proactive distribution company, while the lower-level expresses the profit maximization per rational aggregator. The proposed model is then transformed into a solvable mathematical program with equilibrium constraints through a primal-dual approach. A modified 33-bus distribution network is utilized to demonstrate the effectiveness of the proposed model.
\end{abstract}

Index Terms-Demand response (DR), rational aggregator (RA), proactive distribution company (PDISCO), multi-period AC power flow, mathematical program with equilibrium constraints (MPEC), mathematical program with primal and dual constraints (MPPDC).

\section{NOMENCLATURE}

Sets and Indices

\begin{tabular}{|c|c|}
\hline $\mathcal{N}$ & Set of system buses, indexed by $i$ or $j$. \\
\hline $\mathcal{B}$ & Set of distribution feeders, indexed by $i j$. \\
\hline $\mathcal{K}$ & Set of RAs, indexed by $k$. \\
\hline $\mathcal{L}$ & Set of demands, indexed by $l$. \\
\hline $\mathcal{T}$ & $\begin{array}{l}\text { Set of time periods (e.g., hours per day), } \\
\text { indexed by } t \text {. }\end{array}$ \\
\hline $\mathcal{M}_{l}$ & $\begin{array}{l}\text { Mapping of the set of demands onto the set } \\
\text { of buses. }\end{array}$ \\
\hline $\mathcal{M}_{A g g}$ & $\begin{array}{l}\text { Mapping of the set of demands onto the set } \\
\text { of aggregators. }\end{array}$ \\
\hline
\end{tabular}

The work of C. Zhang and Q. Wang was supported in part by the Danish iPower platform project 10-095378, and in part by the project 255209 from the Research Council of Norway. J. Wang's work was supported by the U.S. Department of Energy (DOE)'s Office of Electricity Delivery and Energy Reliability.

C. Zhang is with the Department of Electric Power Engineering, Norwegian University of Science and Technology, Trondheim, Norway (e-mail: chunyu.zhang@ntnu.no).

Q. Wang, P. Pinson and J. Østergaard are with the Center for Electric Power and Energy, Technical University of Denmark, Kgs. Lyngby, Denmark (e-mail: qiwa@elektro.dtu.dk; ppin@elektro.dtu.dk; joe@elektro.dtu.dk).

J. Wang is with the Energy Systems Division, Argonne National Laboratory, Argonne, IL 60439, USA (e-mail: jianhui.wang@ anl.gov).

J. M. Morales is with the Department of Applied Mathematics and Computer Science, Technical University of Denmark, Kgs. Lyngby, Denmark (e-mail: jmmgo@dtu.dk).

Manuscript received

Variables

$\alpha_{l t}$

$\beta_{l t}$

$P_{k t}^{A g g}, Q_{k t}^{A g g}$

$\lambda_{k t}^{A g g}$

$P_{l t}^{D}, Q_{l t}^{D}$

$Q_{l t}^{D E}$

$\lambda_{i t}^{R D}$

$P_{t}^{R T}, Q_{t}^{R T}$

$P_{l t}^{L S}, Q_{l t}^{L S}$

$P_{i j t}^{\text {Flow }}, Q_{i j t}^{\text {Flow }}$

$Q_{i t}^{C}$

$V_{i t}, \theta_{i t}$

\section{Parameters}

$P_{t}^{S}, Q_{t}^{S}$

$P_{l t}^{D S I}, P_{l t}^{D S E}$

$Q_{l t}^{D S E}$

$P_{l t}^{D I}$

$P_{\text {Aggmin } / \max }$

$Q_{k t}^{\text {Aggmin } / \max }$

$\lambda_{k t}^{A g g P r o}$

$\lambda_{t}^{S}$
Consumption of elastic portion of demand $l$ at time $t$.

Virtual generation of elastic portion of demand $l$ at time $t$.

Active and reactive power produced by RA $k$ at time $t$.

Marginal price for PDISCO purchasing production from RA $k$ at time $t$.

Real-time active and reactive power consumption of demand $l$ at time $t$.

Reactive power output along with virtual generation at demand $l$ at time $t$.

Locational marginal price (LMP) at bus $i$ at time $t$.

Active and reactive power exchanging in real-time market at time $t$.

Active and reactive power of load-shedding at demand $l$ at time $t$.

Active and reactive power flow through feeder $i j$ at time $t$.

Reactive power produced by shunt compensator at bus $i$ at time $t$.

Voltage magnitude and phase angle at bus $i$ at time $t$.

Active and reactive power purchased from day-ahead market at time $t$.

Active power purchased from day-ahead market for inelastic and elastic portions of demand at bus $l$ at time $t$.

Reactive power purchased from day-ahead market for elastic portion of demand at bus $l$ at time $t$.

Real-time inelastic portion of demand at bus $l$ at time $t$.

Active power production bounds of RA $k$.

Reactive power production bounds of RA $k$. Contract price between demand and RA $k$ at time $t$.

Day-ahead market price at time $t$. 


\begin{tabular}{|c|c|}
\hline$\lambda_{t}^{R T}$ & $\begin{array}{l}\text { Real-time price in the real-time market at } \\
\text { time } t \text {. }\end{array}$ \\
\hline$\lambda_{t}^{L S}$ & $\begin{array}{l}\text { Load-shedding penalty price for PDISCO } \\
\text { operation at time } t \text {. }\end{array}$ \\
\hline$\lambda^{D}$ & $\begin{array}{l}\text { Electricity sales price to the demand from } \\
\text { PDISCO. }\end{array}$ \\
\hline$Q_{i}^{C \min / \max }$ & $\begin{array}{l}\text { Reactive power limits of shunt compensator } \\
\text { at bus } i \text {. }\end{array}$ \\
\hline$V_{i}^{\min / \max }$ & Limits of voltage magnitude. \\
\hline $\bar{S}$ & Capacity limit of main substation. \\
\hline$S_{i j}^{\text {Flow }}$ & Capacity limit of feeder $i j$ \\
\hline$G_{i j}, B_{i j}, b_{i j}$ & $\begin{array}{l}\text { Conductance, susceptance and charging sus- } \\
\text { ceptance of feeder } i j \text {. }\end{array}$ \\
\hline$\tau_{i}$ & Transformer tap ratio. \\
\hline$\Gamma$ & Elasticity limit of real-time demand. \\
\hline$\zeta^{\min / \max }$ & Bounds of consumption control factor. \\
\hline & Profit guarantee factor of RA $k$. \\
\hline 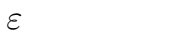 & Inelasticity control factor of each demand. \\
\hline
\end{tabular}

\section{INTRODUCTION}

\section{A. Aim and Approach}

W ITH the increasing penetration and deployment of distributed resources such as demand response (DR), distributed generation (DG), storage devices, etc., distribution systems have become more active than the traditionally passive networks. Distribution companies (DISCOs), as load serving entities (LSEs), have started to get more engaged in electricity market transactions and deal with both market participants in the transmission-level wholesale market and the distributionlevel resources. For example, in the U.S., recent initiatives led by the New York Public Service Commission have addressed regulatory changes to promote utilization of distributed resources mentioned above, such as the New York Reforming Energy Vision (NY REV) [1]. One of the main purposes of NY REV is to establish a distribution-level market platform where all distribution resources can transact and trade with each other, which partly motivates this work. In this paper, we assume the DISCO behaves as a proactive market player, who has the ability to purchase or sell active power according to the real-time market price and conduct bidirectional power exchanging. We propose a modeling framework to capture the procurement strategies of a proactive DISCO, focusing on its interactions with DR aggregators.

As reviewed in [2], DR resources are normally small-size, varied and dispersed, which are difficult to handle systemwide, especially for the real-time operation. To this end, nearly all the applications [3]-[5] and discussions [6], [7] are concentrated on aggregated DR participation in the existing markets at the transmission level, e.g., trading as a virtual plant in the day-ahead market or real-time market. However, derived from the demand side, DR is a natural candidate to directly trade with a DISCO in the local area. Such an advantage may stimulate DR providers to play an essential role in the emerging real-time trading structure, which in turn improves the competitiveness of the market and facilitates the DISCO's electricity procurements among differing resources.

To achieve this goal, at the distribution level, a real-time trading setup is presented along with a newly defined rational aggregator (RA) in this paper. Compared with the small-scale DRs, a RA represents a group of smaller DR resources to bid to the DISCO. Each aggregator concerned in this paper is equipped with certain rationality. In other words, in this competitive environment, each RA tries to fulfil the DISCO's requirement (procurement volumes and offering prices) by rationally putting forward its $\mathrm{kW}$ quantities and bidding prices to maximize its profit. For a DISCO, based on its purchase in the day-ahead market, it has to make optimal decisions on the procurements in the real-time market to adjust its position and maximize its own profit through real-time exchanging, RA virtual generation, possible load-shedding, in addition to electricity sales revenue.

To model load shaving and load shifting, we assume all of the elastic demand is shiftable among the hours. We further divide the elastic demand into shavable and unshavable components, which can capture the DR resource characteristics in a flexible and comprehensive fashion. To the best of our knowledge, no similar DR model in real-time trading has been proposed in the technical literature.

In order to achieve an appropriate trade-off among realtime market transactions, DISCO-RA trading, and others, a multi-period AC power flow formulation is used to accurately represent the underlying physics of the power networks.

Taking into account the listed considerations above, the optimal procurement problem of a proactive DISCO (PDISCO) with RAs can be formulated with a bi-level structure. At each time $t$, the upper-level problem indicates the PDISCO's optimal procurements for maximizing profit, the lower-level problems describe multiple RAs' decisions for rational bidding, one per RA. Note that since the upper-lever problem is non-linear and non-convex due to the involved AC power flow constraints, while the lower-level problems are linear and thus convex, the complexity of this model is increased.

As addressed in [8], this kind of bi-level problem can be transformed into a single-level problem, in which the lower-level problems can be replaced by their first-order optimality conditions. Particularly, in view of the linearity of lower-level RAs' problems, the first-order conditions are formulated by a primal-dual approach, containing primal and dual constraints, and the strong duality requirements. This approach is equivalent to the broadly utilized Karush-KuhnTucker (KKT) conditions, but of high computational efficiency and tractability [8]. Then this reformulated problem renders a mathematical program with equilibrium constraint (MPEC).

\section{B. Literature Review and Contributions}

The literature review is categorized as follows.

1) DISCO procurements: A day-ahead distribution company acquisition market (DCAM) is proposed in [9], in form of the pool market and bilateral contracts. The DISCO purchases electricity according to the offers from DG units, customers, the wholesale market, and contracted load-shedding options. The load, DG units and DCAM objectives are all stipulated in quadratic functions, while the model is just for onehour simulation. Further developed in [10], the DGs and interruptible loads are seen as DRs, and a bi-level model 
is presented. The upper-level represents individual DISCO's profit maximization with its own DGs, the lower-level indicates the day-ahead market clearing model for independent system operator (ISO) to minimize generation costs and loadshedding compensation. However, the DG output and loadshedding price are fixed, and the network constraints are not included. To evaluate the optimal contract pricing between DISCO and DG owners, a bi-level model is also considered in [11], the upper-level objective is to maximize the DG owners' profit (without any physical constraints), the lowerlevel depicts the DISCO network constraints. To avoid the nonconvexity of the constraints, the paper only concerns active power and voltage magnitudes as decision variables, which weaken the effectiveness of the numerical results. A bi-level model is proposed in [12] to investigate the hierarchical market structure between the distribution and transmission networks. The upper-level problem represents the market participant's payoff maximization, and the lower-level problem minimizes the operation cost of the network. However, the locational marginal prices (LMPs) are not endogenously generated by considering the power flow integration. From the management perspective, in [13], a dynamic pricing mechanism is designed to facilitate the aggregator-based DR to participate in the energy schedule of a LSE. A bi-level problem is formulated by taking the LSE as the leader and DR aggregators as the followers. In particular, the DR aggregators are modeled by multi-block utility functions. For simplicity, DC power flow is imposed to model the distribution network, while the impacts of the reactive power are ignored.

2) Real-time DR: The potential interests and feasible applications of real-time DR are illustrated in [14], in which the price uncertainty is accommodated through robust optimization, and the model formulation can be easily applied in a small utility. In [15], a bi-level model is proposed to maximize the retailer's profit and optimize the consumer's behavior under real-time prices. A vital conclusion shows that the real-time pricing is more effective in load shifting. However, this approach depends on a transactional model and lacks network constraints. In contrast, to enable the flexible demand participating in existing electricity markets, a novel pool market mechanism is reported in [16], and further validated by [17]. Although the load shifting is realized by a Lagrangian relaxation based heuristic approach, the network constraints are still not modeled. From the energy management perspective, considering the interactions between DG and the main grid, a contract-based cluster [18] is promoted to initiate DR to purchase or sell energy at a proper time. While this approach also ignores the physical network impacts.

3) Bi-level approach: Recently, the bi-level game structure and complementarity theory have been increasingly adopted in electricity market modeling and analysis, typically reflecting the market outcomes with multiple strategic players competing in the decision-making process. To study the competitive behavior among individual generating companies, an incomplete information bi-level model is proposed in [19]. For strategically controlled microgrids (MGs) [20] in a distribution network, a bi-level model for coordinated operation of the distribution network operator and MGs is presented in [21].
In order to manage multiple MGs, a bi-level model is also proposed in [22] to analyze the competitive situations between an Energy Services Provider (a set of MGs) and a large central production unit. The equilibrium obtained in an oligopolistic electricity pool with network constraints is presented in [23]. DC multi-period power flow is implemented to simplify the transmission network constraints. In the same market setting, to investigate the wind power as a strategic producer, a stochastic bi-level model is proposed in [24]. Pertaining to the DISCO operational issue, a bi-level model is employed on purchasing dispatchable DG and interruptible loads. From the consumer perspective, the authors in [25] proposed an alternative day-head auction scheme for consumer payment minimization in the pool market, while a large consumer procurement strategy is implicitly modeled in [26].

Considering the context above, the main contributions of this paper are threefold:

1) Present a distribution-level trading framework for PDISCO and DR resources.

2) Define a DR formulation for actuating the load shaving and load shifting simultaneously.

3) Propose a methodology for simulating the PDISCO's optimal procurement between real-time power exchanging and RAs' bids, gaming with each RA's bidding in a competitive environment.

\section{Paper Organization}

The rest of this paper is organized as follows. The realtime PDISCO trading structure, DR definition and RA concept are clarified in Section II. Section III proposes the PDISCO's real-time procurement model formulated by a bi-level model, which is further translated into an MPEC with a primal-dual approach. The effectiveness of the proposed methodology is verified by case studies in Section IV. Finally, some relevant conclusions are drawn in Section V.

\section{PDISCO PROCUREMENT}

Traditionally, a DISCO seeks to supply the demands with the lowest possible operation cost. In order to fulfil this goal, the DISCO has to make appropriate decisions on procurement from the day-ahead and real-time electricity markets at the transmission level. Thus, the DISCO is exposed to volatile real-time prices and demand uncertainties. With the availability of DR resources, the DISCO has more flexibility from the demand side. However, small-scale DRs are allocated dispersedly and heterogeneously in the distribution system, which makes it quite difficult to deal with. To address this issue, RA is regarded as a new business player to assemble and schedule the dispersed DR resources.

\section{A. PDISCO}

The aggregator-based DRs can offer the feasibility for prompt load adjustment with superior performance in terms of response time and cost. As shown in Fig. 1, as a profit-driven company, besides supplying the local demands, the DISCO can even execute ambitious schemes to procure excessive DR to 


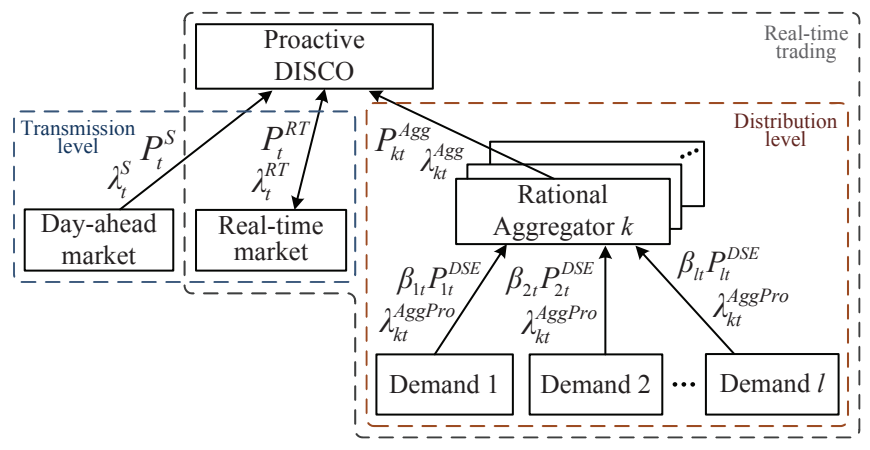

Fig. 1. Real-time procurement framework of the PDISCO.

sell to the real-time market, performing as an active electricity producer, which defines the so-called PDISCO procurement in this paper.

To minimize the payment, the PDISCO has to determine the amounts of electricity purchased from the day-ahead market, transactions with the real-time market and the aggregatorbased DR. Note that, because the PDISCO has thorough knowledge of the day-ahead market prices, the hourly purchases $\left(\lambda_{t}^{S}, P_{t}^{S}\right)$ from the day-ahead market are fixed at the very beginning of the next day. Throughout the day, for each time $t$, the real-time PDISCO trading occurs through the power exchanging (selling or purchasing by $\lambda_{t}^{R T}, P_{t}^{R T}$ ) in the real-time market, and the procurement $\left(\lambda_{k t}^{A g g}, P_{k t}^{A g g}\right)$ with the aggregator-based DR.

Pay-as-bid (PAB) [2] pricing is assumed to be the pricing mechanism between PDISCO and RAs, and one RA is stipulated to submit only one bid at each time $t$. From a DR aggregator perspective, to compete with the others, an aggregator should behave rationally to meet the PDISCO's request, and send the proper bids ( $\mathrm{kW}$ quantity and bidding price) to the PDISCO, implying the target of its own profit maximization. At the same time, according to the available real-time market price, the dynamic active/reactive consumption, RA bids, and possible load-shedding, further constrained by the physical network and facility limits, the PDISCO has to optimize the real-time trading decisions in a complex situation.

Therefore, the interactions between PDISCO and RAs can be characterized by a bi-level game-theoretic model as discussed in Section III.

\section{B. Demand Response}

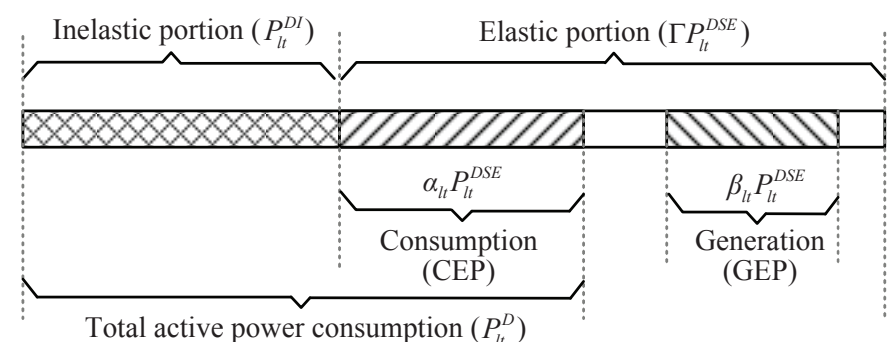

Fig. 2. Definition of demand response.
In this paper, a demand is defined as the summation of both the inelastic portion and elastic portion. In the dayahead market, demand $l$ takes both these portions $\left(P_{l t}^{D S I}\right.$ and $P_{l t}^{D S E}$ ) into account to make an electricity purchase. In realtime operation, as shown in Fig. 2, the inelastic portion $P_{l t}^{D I}$ is the indispensable consumption of demand $l$, and can be deemed as the same quantity as $P_{l t}^{D S I}$. In addition, the elastic portion can be assigned as $\Gamma P_{l t}^{D S E}(\Gamma \geqslant 1)$ and further divided into two parts. The first part (referred to as $\alpha_{l t} P_{l t}^{D S E}$ ) is for the actual consumption of the elastic portion (CEP), which represents the shifting flexibility of real-time demand during time period $t$. This indicates the DR capability of load shifting. The second part (denoted as $\beta_{l t} P_{l t}^{D S E}$ ) expresses the shavable demand, which can be seen as virtual generation of the elastic portion (GEP) managed by a RA and sold to the PDISCO. This implies the DR function of load shaving. Thus, the total active power consumption of demand $l\left(P_{l t}^{D}\right)$ consists of $P_{l t}^{D I}$ and $\alpha_{l t} P_{l t}^{D S E}$.

\section{Rational Aggregator}

At the distribution level, the new defined RA is a virtual business entity, who is independent and has no physical integration with the system network, but has the commercial and technical abilities to behave rationally as follows:

1) For the sake of harvesting DR generations (GEPs), at each time $t$, RA acquires individual GEPs with contracts, and makes an optimal decision on pricing.

2) In the real-time trading process, as a competitive market player, a RA satisfies the PDISCO's request, self-evaluates the availabilities of the contractual DRs, sets up the bidding price and $\mathrm{kW}$ quantity, and bids to the PDISCO effectively.

3) After obtaining offers (procurement volumes and offering prices) from the PDISCO, RA mobilizes the corresponding GEP portfolios to meet the requirements. Besides, the PDISCO also passes the related CEP shifting schedules to the RA on the basis of system requirements, and the latter takes the responsibility of executing the schedule.

\section{PRoblem Formulation}

\section{A. Assumptions}

The mathematical formulation of the proposed PDISCO procurement model is based on the following assumptions.

1) We assume that a PDISCO owns and operates the distribution network, and physically connects to the transmission grid via only one main substation.

2) As for the real-time trading, multi-period AC power flow is adopted to represent the distribution network. We assume only the active power can be traded in the real-time market and between the PDISCO and RAs, since no uniform reactive power market has evolved.

3) In the real-time market, when the PDISCO is recognized as an active producer, its offering price is assumed to be the marginal price cleared at the interconnection point (main substation) with the transmission system, and its offering volume is based on the surplus of individual RAs' bids after satisfying the distribution network constraints. 
4) Each RA can explicitly predict the impact of its bids (bidding prices and $\mathrm{kW}$ quantities), versus the PDISCO offers (offering prices and procurement volumes). This is reflected as the linking variable $\lambda_{k t}^{A g g}$ within this bi-level model.

5) For simplicity, regarding each RA's demand contract, all the related demands are assumed to be paid with an identical price.

\section{B. Bi-level Model}

Note that the upper-level and the lower-level problems are interrelated with each other. The bid price and quantity, put forward by the RAs from lower-level problems, impact the PDISCO's procurement decisions in the upper-level. On the other hand, the upper-level problem determines the offering price and procurement volume, which directly influence the RAs' profits in the lower-level problems. Therefore, the formulation of the proposed bi-level model is made up of two optimization levels, i.e. the upper-level (1)-(24) is for PDISCO procurement decisions, and the lower-level (25)-(30) is for the rational bidding of each $\mathrm{RA}$.

$$
\begin{aligned}
& \Xi^{A g g \cup \Xi^{P D I S C O} \cup \Xi^{D u a l}}\left(\sum_{t, k} \lambda_{k t}^{A g g} P_{k t}^{A g g}+\sum_{t} \lambda_{t}^{R T} P_{t}^{R T}\right. \\
& \left.+\sum_{t} \lambda_{t}^{S} P_{t}^{S}+\sum_{t, l} \lambda_{t}^{L S} P_{l t}^{L S}-\lambda^{D} \sum_{t, l} P_{l t}^{D}\right) \\
& \text { s.t. } \\
& 0 \leq \alpha_{l t}+\beta_{l t} \leq \Gamma, \forall l, t \\
& P_{l t}^{D I}=P_{l t}^{D S}, \forall l, t \\
& P_{l t}^{D}=P_{l t}^{D I}+\alpha_{l t} P_{l t}^{D S E}, \forall l, t \\
& \delta_{k t} \lambda_{k t}^{A g g P r o} \leq \lambda_{k t}^{A g g} \leq \lambda_{t}^{R T}, \forall k, t \\
& \text { For the main substation }(\text { reference bus): } \\
& P_{t}^{S}+P_{t}^{R T}+\beta_{1 t} P_{1 t}^{D S E}+P_{1 t}^{L S}-P_{1 t}^{D} \\
& =\sum_{1 j, j \neq 1} P_{1 j t}^{F l o w}, \forall t \\
& Q_{t}^{S}+Q_{t}^{R T}+Q_{1 t}^{C}+Q_{1 t}^{D E}+Q_{1 t}^{L S}-Q_{1 t}^{D} \\
& =\sum_{1 j, j \neq 1} Q_{1 j t}^{F l o w}, \forall t \\
& \theta_{1 t}=0 \\
& V_{1 t}=1, \\
& \left(P_{t}^{S}+P_{t}^{R T}\right)^{2}+\left(Q_{t}^{S}+Q_{t}^{R T}\right)^{2} \leq \bar{S}^{2}, \forall t \\
& \text { For the other buses: }
\end{aligned}
$$

For the other buses:

$$
\begin{aligned}
& \beta_{l t} P_{l t}^{D S E}+P_{l t}^{L S}-P_{l t}^{D}=\sum_{i j, j \neq i} P_{i j t}^{F l o w},: \lambda_{i t}^{R D} \\
& \forall i, t, l:(l, i) \in \mathcal{M}_{l} \\
& Q_{i t}^{C}+Q_{l t}^{D E}+Q_{l t}^{L S}-Q_{l t}^{D}=\sum_{i j, j \neq i} Q_{i j t}^{F l o w}, \\
& \forall i, t, l:(l, i) \in \mathcal{M}_{l} \\
& P_{i j t}^{F l o w}=-\tau_{i} V_{i t}^{2} G_{i j}+V_{i t} V_{j t}\left[G_{i j} \cos \left(\theta_{i t}-\theta_{j t}\right)\right. \\
& \left.+B_{i j} \sin \left(\theta_{i t}-\theta_{j t}\right)\right], \forall i, j, t \\
& Q_{i j t}^{F l o w}=\tau_{i} V_{i t}^{2} B_{i j}-0.5 b_{i j}+V_{i t} V_{j t}\left[G_{i j} \sin \left(\theta_{i t}-\theta_{j t}\right)\right.
\end{aligned}
$$

level problem variables. $\Xi^{P D I S C O}=\left\{\alpha_{l t}, \lambda_{k t}^{A g g}, P_{l t}^{D}, Q_{l t}^{D}\right.$, $\left.Q_{l t}^{D E}, P_{l t}^{L S}, Q_{l t}^{L S}, P_{t}^{R T}, Q_{t}^{R T}, P_{i j t}^{\text {Flow }}, Q_{i j t}^{\text {Flow }}, Q_{i t}^{C}, V_{i t}, \theta_{i t}\right\}$ is the set of upper-level problem variables. $\Xi^{\text {Dual }}=\left\{\lambda_{i t}^{R D}, \eta_{k t}\right.$, $\left.\mu_{k t}, \sigma_{k t}^{-}, \sigma_{k t}^{+}, \rho_{k t}^{-}, \rho_{k t}^{+}, \phi_{l t}^{-}, \phi_{l t}^{+}\right\}$is the set of dual variables.

The objective (1) of the upper-level problem is to minimize the PDISCO's minus-profit, which comprises the cost of purchasing the GEPs from RAs, exchanging power from the real-time market, acquiring active power from the dayahead market, the penalty of possible load-shedding, and the minus revenue of electricity sales to demands. For each time $t$ : Constraints (2) enforce the bounds of the GEP/CEP produced/consumed by each demand. Constraints (3) impose the real-time quantity of inelastic active power is the same as purchased from the day-ahead market. Furthermore, constraints (4) indicate the consumed active power is composed of the inelastic portion $P_{l t}^{D I}$ and the CEP portion $\alpha_{l t} P_{l t}^{D S E}$. From the perspective of the PDISCO, associated with the bid control

where $\Xi^{A g g}=\left\{P_{k t}^{A g g}, Q_{k t}^{A g g}, \beta_{l t}\right\}$ is the set of each lower$\left.-B_{i j} \cos \left(\theta_{i t}-\theta_{j t}\right)\right], \forall i, j, t$
$-\pi \leq \theta_{i t} \leq \pi, \forall i, t$ $V_{i}^{\text {min }} \leq V_{i t} \leq V_{i}^{\max }, \forall i, t$
$\left(P_{i j t}^{\text {Flow }}\right)^{2}+\left(Q_{i j t}^{\text {Flow }}\right)^{2} \leq\left(S_{i j}^{\text {Flow }}\right)^{2}, \forall i, j, t$ $Q_{i}^{C \min } \leq Q_{i t}^{C} \leq Q_{i}^{C \max }, \forall i, t$
$0 \leq P^{L S} \leq P^{D}$

$P_{l t}^{L S} Q_{l t}^{D}=P_{l t}^{D} Q_{l t}^{L S}, \forall l, t$

$\sum P_{l t}^{D}-\sum P_{l t}^{L S} \geq \zeta^{\text {min }} \sum P_{t}^{S}$

$\sum_{l, t} P_{l t}^{D}-\sum_{l, t} P_{l t}^{L S} \leq \zeta^{\max } \sum_{t} P_{t}^{S}$

$P_{k t}^{A g g m i n} \leq P_{k t}^{A g g} \leq P_{k t}^{\text {Aggmax }}, \forall k, t: \rho_{k t}^{-}, \rho_{k t}^{+}$

$Q_{k t}^{A g g m i n} \leq Q_{k t}^{A g g} \leq Q_{k t}^{A g g m a x}, \forall k, t: \sigma_{k t}^{-}, \sigma_{k t}^{+}$

$\left.0 \leq \beta_{l t} \leq 1, \forall t, l:(l, k) \in \mathcal{M}_{A g g}: \phi_{l t}^{-}, \phi_{l t}^{+}\right\}$

$\alpha_{l t}, \beta_{l t}, P_{k t}^{A g g}, Q_{k t}^{A g g}, \lambda_{k t}^{A g g}, P_{l t}^{D}, Q_{l t}^{D}, Q_{l t}^{D E}$, $\lambda_{i t}^{R D}, P_{l t}^{L S}, Q_{l t}^{L S}, Q_{l t}^{C}, V_{i t}, \sigma_{k t}^{-}, \sigma_{k t}^{+}, \rho_{k t}^{-}, \rho_{k t}^{+}$,

$\phi_{l t}^{-}, \phi_{l t}^{+} \geq 0$

$P_{t}^{R T}, Q_{t}^{R T}, P_{i j t}^{\text {Flow }}, Q_{i j t}^{\text {Flow }}, \theta_{i t}, \eta_{k t}, \mu_{k t}$ : free. (1)

(1)

(1)

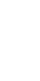


factor $\delta_{k t}\left(\delta_{k t} \geq 1, \forall k, t\right)$, a RA profit guarantee mechanism is yielded as the lower bound of constraints (5), which also emphasizes the acceptable RA's bidding price should be no greater than the price from the real-time market (ceiling price). Here, AC power flow is employed to formulate the realtime operation model. For the main substation (reference bus), constraints (6) and (7) represent the AC power balance, and the voltage angle and voltage value are retained at constant level via constraints (8) and (9). The capacity limit of the main substation is specified in constraints (10). For the other buses, constraints (11), (12), (13) and (14) identify the AC power flowing through the feeder $i-j$, and constraints (17) further impose the capacity limits individually. Constraints (15) and (16) identify the angle bounds and voltage limits for the other buses. Constraints (18) describe the capacity bounds for each compensator. Specifically for the potential load-shedding bus, the amount of load curtailment invoked by the PDISCO is capped with the constraints (19). Constraints (20) express the limits of the elastic reactive power for each demand. Constraints (21) and (22) keep the power factor in constant, if the corresponding demand is involved in load-shedding or GEP-generating. Constraints (23) and (24) state consumption control over the whole timespan (e.g., 24 hours per day) with the bounds $\zeta^{\min / \max }$. For instance, when $\zeta^{\min }=\zeta^{\max }=1$, these constrains guarantee the consumption of each demand across the whole timespan should be equal to the same amount purchased by the PDISCO from the day-ahead market. That means, these constraints ensure the load shifting between the hours while maintaining the total consumption at a certain level.

As indicated in (25), the objective of the lower-level problem is to minimize the minus-profit of each RA $k$, i.e. the cost of purchasing GEPs from contractual demands minus the revenue of selling the aggregated quantities to the PDISCO, correspondingly. Observe that the PDISCO offering price $\lambda_{k t}^{A g g}$ is an upper-level decision variable treated as a parameter within the lower-level problem. This means that once the RAs rationally submit their bidding prices $\lambda_{k t}^{A g g}$ with $\mathrm{kW}$ quantities $P_{k t}^{A g g}$, the PDISCO figures out the preferable offers through the upper-lever problem. Constraints (26) and (27) illustrate the valid GEPs (active power) with the essential reactive power $Q_{l t}^{D E}$ assembled by each RA, while the output limits on individuals are imposed by constraints (28) and (29). Constraints (30) preserve the GEPs of each demand ought to be less than the amount $P_{l t}^{D S E}$ purchased from the day-ahead market.

Finally, constraints (31) classify the positive variables and free variables for this bi-level model.

\section{C. $M P E C$}

In this subsection, the bi-level model for the procurement decision-making of the PDISCO with RAs is transformed into a single-level optimization problem. Since each lower-level problem is linear and thus convex, its Karush-Kuhn-Tucker (KKT) optimality conditions are necessary and sufficient. However, the complementarity derived from KKT conditions is numerically difficult to be handled especially when the upper-level PDISCO problem is already non-linear and nonconvex. Therefore, the KKT approach is not appropriate to solve this bi-level problem tractably. To avoid complementarity conditions, the Primal-dual approach [8], [27] is applied in this paper, rendering a mathematical program with primal and dual constraints (MPPDC).

Constraints (32)-(34) are dual constraints of lower-level RA problems. The constraint (35) is the associated strong duality equality, which ensures the equality of the primal and dual objective function values, one per RA $k$.

Dual constraints:

$$
\begin{aligned}
& \lambda_{k t}^{\text {AggPro }}-\lambda_{k t}^{A g g}+\eta_{k t}+\rho_{k t}^{+}-\rho_{k t}^{-}=0, \forall k, t \\
& \mu_{k t}+\sigma_{k t}^{+}-\sigma_{k t}^{-}=0, \forall k, t \\
& -\eta_{k t} P_{l t}^{D S E}+\phi_{l t}^{+}-\phi_{l t}^{-}=0, \forall l:(l, k) \in \mathcal{M}_{\text {Agg }}, t
\end{aligned}
$$

Strong duality equality:

$$
\begin{aligned}
& \sum_{t}\left(\lambda_{k t}^{\text {AggPro }} P_{k t}^{A g g}-\lambda_{k t}^{\text {Agg }} P_{k t}^{\text {Agg }}\right) \\
& +\sum_{t}\left(\mu_{k t} \sum_{l:(l, k) \in \mathcal{M}_{A g g}} Q_{l t}^{D E}\right)+\sum_{t}\left(P_{k t}^{\text {Aggmax }} \rho_{k t}^{+}\right. \\
& \left.-P_{k t}^{\text {Aggmin }} \rho_{k t}^{-}+Q_{k t}^{\text {Aggmax }} \sigma_{k t}^{+}-Q_{k t}^{\text {Aggmin }} \sigma_{k t}^{-}\right) \\
& +\sum_{t, l:(l, k) \in \mathcal{M}_{A g g}} \phi_{l t}^{+}=0, \forall k
\end{aligned}
$$

Substituting lower-level RA problems with MPPDC, the final single-level model is to minimize the PDISCO's objective, subject to PDISCO's constraints, RAs' MPPDC constraints, and declarations of positive and free variables, as shown in Fig. 3. The final non-linear model without complementarity can be solved by the commercial off-the-shell large-scale non-linear optimization solver CONOPT3 [28].

$$
\begin{aligned}
& \underset{\Xi^{A g g} \cup \Xi^{P D I S C O} \cup \Xi^{D \text { ual }}}{\text { Minimize }}(1) \\
& \text { s.t. } \\
& \text { PDISCO's problem constraints: } \\
& (2)-(24) \\
& \text { RAs' problems MPPDC constraints: } \\
& \text { (26) - (30) and (32) - (35) } \\
& \text { Variables Declarations: }
\end{aligned}
$$

(31)

Fig. 3. The reformulated single-level model.

\section{Case Studies}

In this section, a 33-bus distribution network [29] is modified to validate the effectiveness of the methodology proposed in Section III. All cases are solved by CONOPT3 with GAMS 24.4.1 [28] on a 3.6 GHz Intel Core i7 processor carried out on $16 \mathrm{~GB}$ of RAM and 64-bit Windows 7 system.

\section{A. Data}

The 33-bus distribution network presented in [29] is considered to be owned and operated by the PDISCO. The topology, 
feeder capacity, and impedance parameters are shown in this section. Capacity of each feeder $S_{i j}^{F l o w}$ is set to $10 \mathrm{MVA}$. The main substation capacity limit $S$ is imposed to 20 MVA. At the reference bus, the voltage is 1 p.u. with the voltage angle of 0 . The tap ratio $\tau_{i}$ per transformer is fixed to 1 . For the other buses, the bounds of voltage $\left(V_{i}^{\min / \max }\right)$ are 0.9 and 1.1 p.u.. Each compensator's output is enforced in the range of 0-200 kVar. To address the inelastic portion per demand, an inelasticity control factor $\varepsilon$ is adopted to specify this proportion, i.e. $P_{l t}^{D S I}=\varepsilon P_{l t}^{S}, P_{l t}^{D S E}=(1-\varepsilon) P_{l t}^{S}$. Three RAs are concerned to be hourly involved in the real-time trading with the PDISCO, i.e. 24 times per day. The individual zoning is initialed as, RA1 includes $\{2,3,4,5,6,7,19,20,21,23,26\}$, RA2 contains $\{10,11,12,13,14,15,16,17,18,24,25\}$, and RA3 consists of $\{8,9,22,27,28,29,30,31,32,33\}$. Referring to the NordPool [30] prices, the day-ahead market price $\lambda_{t}^{S}$ and real-time market price $\lambda_{t}^{R T}$ can be estimated and given in Table I-II, which also enumerate the day-ahead market volume $P_{t}^{S}$, RA-demand contract price $\lambda_{k t}^{A g g P r o}$, and load-shedding price $\lambda_{t}^{L S}$.

For simplicity, the profit guarantee factor $\delta_{k t}$ held by each RA $k$ is identical and equal to 1.1. Other system-wide parameters are imposed as: the demand purchase price $\lambda^{D}=0.6$ $€ / \mathrm{kW}$, elasticity limit $\Gamma=1.2$, daily consumption control factor $\zeta^{\min }=\zeta^{\max }=1$.

TABLE I

PARAMETERS OF DAY-AHEAD AND REAL-TIME MARKETS

\begin{tabular}{c|c|c|c}
\hline $\begin{array}{c}\text { Time } \\
t \\
{[\text { Hour }]}\end{array}$ & $\begin{array}{c}\text { Real-time } \\
\text { market price } \\
\lambda_{t}^{R T} \\
{[€ / \mathrm{kW}]}\end{array}$ & $\begin{array}{c}\text { Day-ahead } \\
\text { market price } \\
\lambda_{t}^{S} \\
{[€ / \mathrm{kW}]}\end{array}$ & $\begin{array}{c}\text { Day-ahead } \\
\text { market transaction } \\
P_{t}^{S} \\
{[\mathrm{~kW}]}\end{array}$ \\
\hline 1 & 0.13 & 0.11 & 1114.50 \\
\hline 2 & 0.12 & 0.10 & 1114.50 \\
\hline 3 & 0.15 & 0.12 & 1300.25 \\
\hline 4 & 0.11 & 0.10 & 1114.50 \\
\hline 5 & 0.30 & 0.26 & 2972.00 \\
\hline 6 & 0.32 & 0.27 & 2972.00 \\
\hline 7 & 0.35 & 0.29 & 3343.50 \\
\hline 8 & 0.40 & 0.30 & 3715.00 \\
\hline 9 & 0.42 & 0.31 & 3715.00 \\
\hline 10 & 0.66 & 0.58 & 6315.50 \\
\hline 11 & 0.71 & 0.62 & 6687.00 \\
\hline 12 & 0.74 & 0.64 & 6687.00 \\
\hline 13 & 0.69 & 0.59 & 6315.50 \\
\hline 14 & 0.50 & 0.38 & 3715.00 \\
\hline 15 & 0.41 & 0.31 & 3715.00 \\
\hline 16 & 0.40 & 0.30 & 3715.00 \\
\hline 17 & 0.42 & 0.31 & 3715.00 \\
\hline 18 & 0.60 & 0.53 & 5572.50 \\
\hline 19 & 0.65 & 0.55 & 5944.00 \\
\hline 20 & 0.67 & 0.56 & 6315.50 \\
\hline 21 & 0.70 & 0.60 & 6501.25 \\
\hline 22 & 0.35 & 0.27 & 2972.00 \\
\hline 23 & 0.28 & 0.24 & 1857.50 \\
\hline 24 & 0.15 & 0.12 & 1486.00 \\
\hline
\end{tabular}

\section{B. PDISCO Procurement and RAs' Profits}

The results of the PDISCO procurement are shown in Fig. 4 (a)-(b). As expected, during high-price hours (10-14, 1822) in the real-time market, the PDISCO performs as an active producer. In the other time slots, PDISCO acquires very little from RAs, who executes demand shifting according to shifting schedules passed by PDISCO. Therefore, the demand
TABLE II

PARAMETERS FOR RAS AND DEMANDS

\begin{tabular}{|c|c|c|c|c|}
\hline $\begin{array}{c}\text { Time } \\
t \\
{[\text { Hour }]}\end{array}$ & $\begin{array}{c}\text { RA1-demand } \\
\text { contract price } \\
\lambda_{1 t}^{\text {AggPro }} \\
{[€ / \mathrm{kW}]}\end{array}$ & $\begin{array}{c}\text { RA2-demand } \\
\text { contract price } \\
\lambda_{2 t}^{A g g P r o} \\
{[€ / \mathrm{kW}]}\end{array}$ & $\begin{array}{c}\text { RA3-demand } \\
\text { contract price } \\
\lambda_{3 t}^{\text {AggPro }} \\
{[€ / \mathrm{kW}]}\end{array}$ & $\begin{array}{c}\text { Load- } \\
\text { shedding price } \\
\lambda_{t}^{L S} \\
{[€ / \mathrm{kW}]}\end{array}$ \\
\hline 1 & 0.05 & 0.08 & 0.06 & 8.00 \\
\hline 2 & 0.05 & 0.08 & 0.07 & 8.00 \\
\hline 3 & 0.05 & 0.09 & 0.07 & 9.00 \\
\hline 4 & 0.04 & 0.07 & 0.05 & 7.00 \\
\hline 5 & 0.11 & 0.18 & 0.15 & 16.00 \\
\hline 6 & 0.12 & 0.20 & 0.16 & 17.00 \\
\hline 7 & 0.13 & 0.22 & 0.17 & 18.00 \\
\hline 8 & 0.15 & 0.24 & 0.19 & 20.00 \\
\hline 9 & 0.16 & 0.25 & 0.20 & 21.00 \\
\hline 10 & 0.24 & 0.41 & 0.33 & 35.00 \\
\hline 11 & 0.26 & 0.42 & 0.36 & 36.00 \\
\hline 12 & 0.28 & 0.43 & 0.37 & 37.00 \\
\hline 13 & 0.25 & 0.40 & 0.32 & 34.00 \\
\hline 14 & 0.18 & 0.26 & 0.21 & 22.00 \\
\hline 15 & 0.15 & 0.24 & 0.20 & 20.00 \\
\hline 16 & 0.14 & 0.22 & 0.18 & 20.00 \\
\hline 17 & 0.15 & 0.25 & 0.19 & 20.00 \\
\hline 18 & 0.20 & 0.36 & 0.30 & 31.00 \\
\hline 19 & 0.21 & 0.36 & 0.29 & 32.00 \\
\hline 20 & 0.22 & 0.41 & 0.30 & 33.00 \\
\hline 21 & 0.24 & 0.42 & 0.33 & 35.00 \\
\hline 22 & 0.12 & 0.22 & 0.16 & 17.00 \\
\hline 23 & 0.11 & 0.19 & 0.15 & 16.00 \\
\hline 24 & 0.06 & 0.09 & 0.07 & 8.00 \\
\hline
\end{tabular}

consumes more in the low-price periods, and results in a more rational load curve. Furthermore, to avoid an extremely high penalty price, load-shedding is not used at any bus.

Regarding the PDISCO's profit, we can observe that the maximum income is not placed during hours $10-14$ or 18-22, but spread out in other time periods. That is because PDISCORA trading shaves high-price peak load and shifts it to the other hours, while the daily consumption remains unchanged and the electricity purchase price $\lambda^{D}$ by the demand side is fixed. Thus, the PDISCO daily profit can reach $€ 17958.97$.

Subsequently, the profit results of individual RAs are also shown in Fig. 4 (b). At each time $t$, each RA rationally bids at the equilibrium price and quantity, and dispatches individual contractual demands. The daily profits of RA 1, RA2 and RA3 are about $€ 72.59$, €164.83 and $€ 112.45$, respectively.

\section{Impact of Elasticity Limit $\Gamma$}

As indicated in Section III, the elasticity limit $\Gamma$ enforces the the upper bound of GEP/CEP produced/consumed by each demand $l$ via constraint (2). Thus, $\Gamma$ is the key factor impacting the real-time demand consumption, PDISCO procurement decision, RA bidding, and demand revenue, simultaneously. Keeping the other parameters unchanged, given the increment of $\Gamma$ with 0.1 per interval, as shown in Fig. 5 (a), the PDISCO's daily profit, RAs' profits and demand revenue keep increasing rapidly, but tend to be flat when $\Gamma$ goes beyond 1.4. That implies, on the basis of system operation constraints, the PDISCO has to find a trade-off among exchanging with the real-time market, procuring RAs' virtual generation, and maintaining daily consumption at a critical level. On the other side, extra availability of GEPs causes a more completive market, some RAs (e.g., RA1 and RA2) may reduce their interests in trading with PDISCO. 


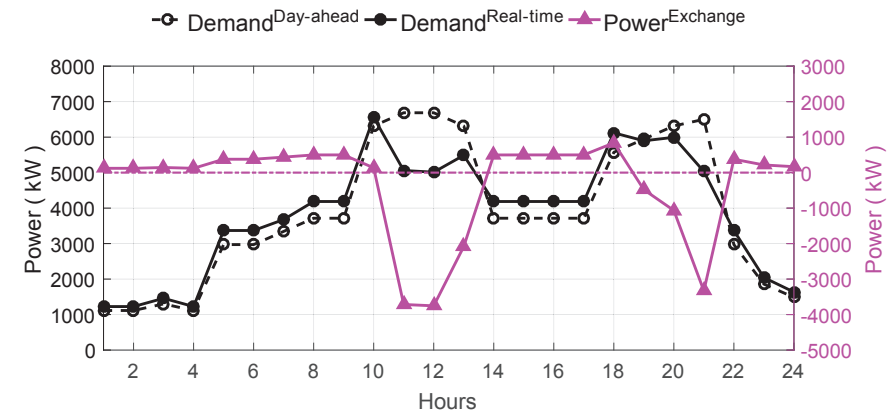

(a)

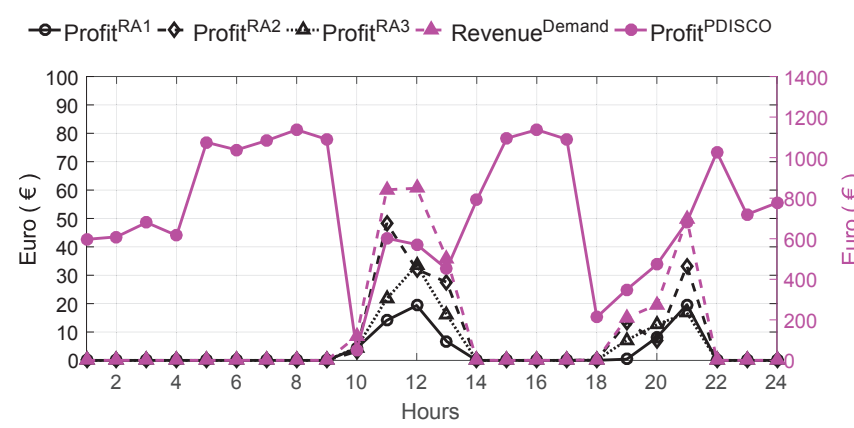

(b)

Fig. 4. Results of the PDISCO procurement and RAs' profits.

\section{Impact of Inelasticity Control Factor $\varepsilon$}

The inelasticity control factor $\varepsilon$ directly reflects the consumption characteristic, and indirectly affects the GEP per demand, as illustrated in Section II-B. With the other parameters unchanged, Fig. 5 (b) depicts the relations of daily PDISCO profit, RA profit and demand revenue under differing $\varepsilon$ values. Observe that, with the $\varepsilon$ increasing, the diminishing elasticity results in limited trading possibilities among the participants.

\section{E. Impact of Daily Consumption Control Factor $\zeta^{\min / \max }$}

To embody the sensitivity of daily consumption adopted in this proposed bi-level model, we categorize the control factor $\zeta^{\min / \max }$ into three cases. With the other parameters unchanged, the results are shown in Fig. 5 (c).

1) Case 1: $\zeta^{\min } \geqslant 1, \zeta^{\max } \geqslant 1$. Compared with $\zeta$ allocated in the range of [1-1.1], each market participant obtains slightly more profit if the daily consumption is strictly controlled at $\zeta^{\min }=\zeta^{\max }=1$. This indicates the PDISCO intends to evade demand-side risks and hope consumption at each bus can be realized as the same amount as purchased from the day-ahead market.

2) Case 2: $\zeta^{\min }<1, \zeta^{\max }>1$. In this situation, this proposed trading setup allows moderate arbitrage to have both PDISCO and RAs to pursue higher profit. Compared with case 1, we set $\zeta$ in [0.9-1.1] and [0.9-1]. Both of the results show that, a proper bound of consumption control can increase around $15 \%$ of the daily profit for the PDISCO, and critically prompt $75 \%-87 \%$ of profit for individual RAs. Thus, the demand obtains $81 \%$ extra revenues.

3) Case 3: $\zeta^{\min }<1, \zeta^{\max }<1$. With the tendency of exacerbating arbitrage behavior, i.e. $\zeta$ is arranged in [0.8-0.9] and [0.7-0.8], the RAs' profits persist with a rising trend. On the contrary, the PDISCO's daily profit drops rapidly compared to case 2 , and a reduction of $23 \%$ from case 1 .

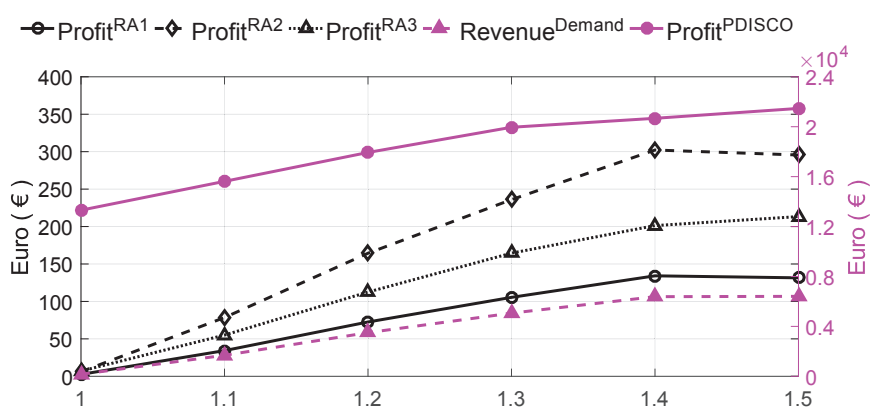

(a) $\Gamma$

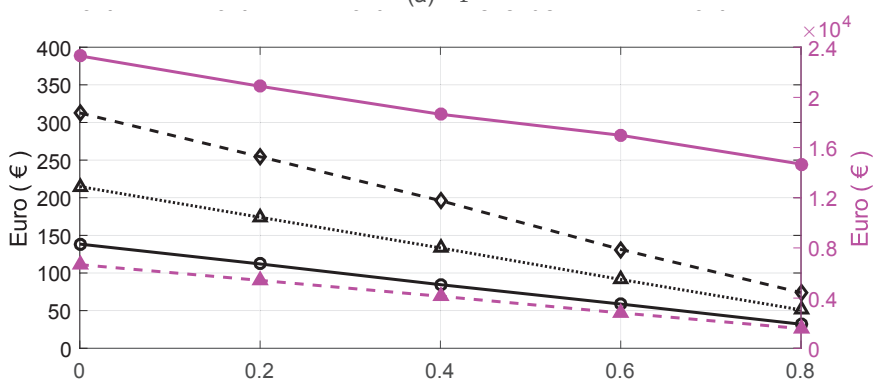

(b) $\epsilon$

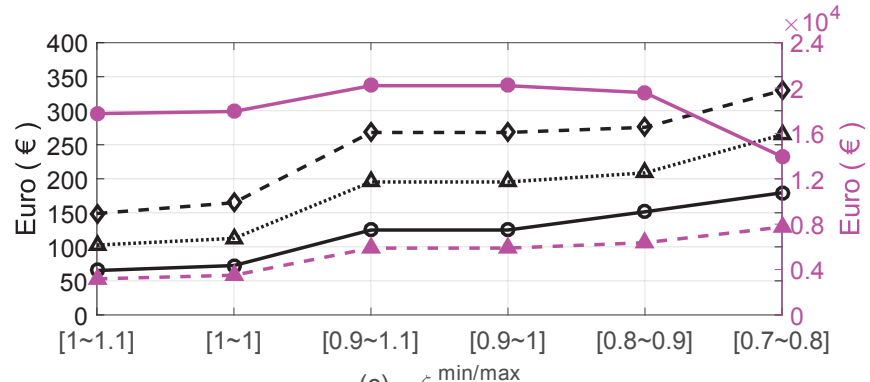

(c) $\zeta^{\min / \max }$

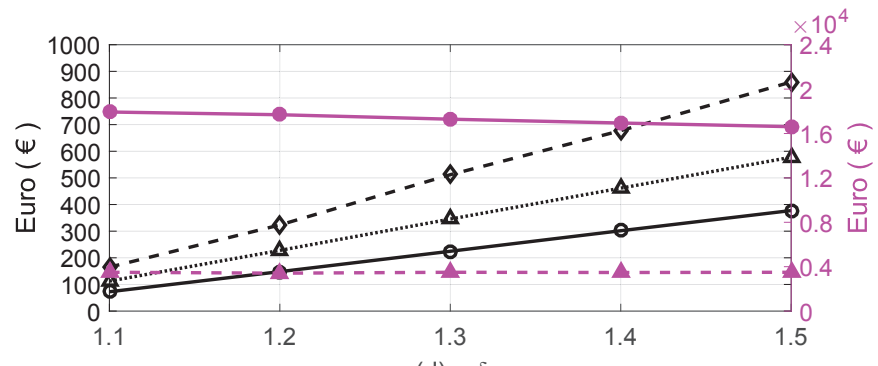

(d) $\delta$

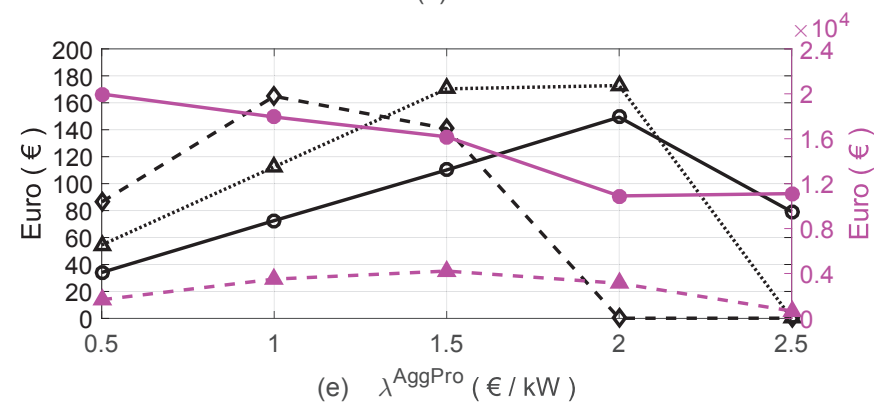

Fig. 5. Impacts of individual sensitive parameters.

Therefore, the moderate arbitrage strategy may be more preferable for the PDISCO to maximize profit, also for RAs 
to trade with the PDISCO rationally.

\section{F. Impact of Profit Guarantee Factor $\delta_{k t}$}

Aiming to be a profitable participant gaming with the PDISCO, the profit guarantee factor $\delta_{k t}$ represents the expected price margin of RA $k$. As shown in Fig. 5 (d), increasing $\delta_{k t}$ to 1.5 with 0.1 increments, the RAs' profits rise up sharply, while the PDISCO's daily profit decreases progressively and the demand revenue stays at a certain level. On one hand, this validates the PDISCO is willing to continuously procure RAs' virtual generation under the premise that the bidding prices $\lambda_{k t}^{A g g}$ are comparable to the real-time price $\lambda_{t}^{R T}$. On the other hand, to maximize RA's own profit, the procurement price $\lambda_{k t}^{A g g P r o}$ should be optimized with the contractual GEP providers.

\section{G. Impact of $R A$ Procurement Price $\lambda_{k t}^{\text {AggPro }}$}

To capture the characteristics of RA procurement price $\lambda_{k t}^{\text {AggPro}}$, we assume the data in IV-A represents a base case and impose a $\lambda_{k t}^{A g g P r o}$ multiplier from [0.5-2.5]. Fig. 5 (e) indicates the PDISCO's daily profit declines gradually, while each RA has varied performance. For RA1 and RA3, their profits are maximized at multiplier $=2$ and go down immediately afterwards, since their bidding prices are approaching the price ceiling $\lambda_{k t}^{R T}$. Prior to that point, a similar observation can be obtained from RA2. Accordingly, the PDISCO's profit has been reduced significantly at each time when a RA quits realtime trading. When multiplier=2.5, RA1 is left as the single player to trade with PDISCO. In this case, the PDISCO-RA trading turns into a monopolistic structure.

\section{$H$. Impact of Number of RAs}

In the proposed approach, associated with a certain number of RAs, the PDISCO makes optimal decisions on electricity procurements. To test the PDISCO's profitability through multiple RA combinations, $\kappa$ is used to distinguish the number of RAs, and the results are shown in Fig. 6.

1) $\kappa=1$ : As a base case, if no RA exists in this PDISCORA trading model, the daily PDISCO profit is $€ 12938.88$. Particularly, RA1, RA2 and RA3 respectively represent the participants with the low, high, and medium prices involved in the PDISCO-RA trading process. As mentioned above, a single RA interacting with the PDISCO is in a monopolistic setting. We can observe that the RA's bidding price goes higher while the PDISCO's profit gets lower, e.g., RA1 bids with $\lambda_{1 t}^{A g g P r o}$ and the PDISCO gains $€ 21651.20$ daily profit, in contrast, RA2 bids with $\lambda_{2 t}^{A g g P r o}$ and the PDISCO's profit reduces to $€ 19930.40$.

2) $\kappa=2$ : The observation mentioned above can also be obtained in the two-RA case, which denotes a moderately competitive market. The PDISCO's profit shows a reverse trend with the RA's bidding prices, and the lowest profit ( $€$ 18543.44) appears in the existence of RA2 and RA3.

3) $\kappa=3$ : In a more competitive market case, the PDISCO claims more RA virtual generation, and three rival RAs compete with each other on bidding prices and quantities to

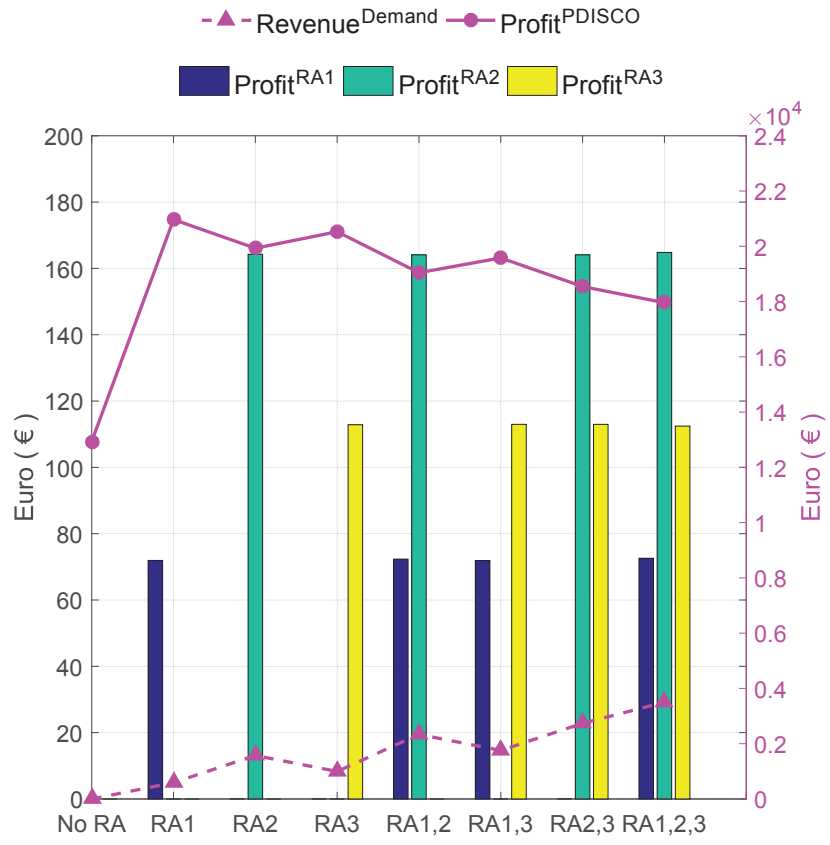

Fig. 6. Impact of integrated RA number $\kappa$.

maximize their own profit. Contrarily, the PDISCO's profit further declines to $€ 17958.97$, but still shows $39 \%$ higher than the non-RA case.

Among these cases, the demand revenue grows gradually along with the increasing number $\kappa$ of RAs, and presents an opposite trend against the PDISCO's profit.

TABLE III

COMPUtational Time PeR Number $\kappa$

\begin{tabular}{c|c|c}
\hline Number & RA & CUP time (s) \\
\hline \multirow{3}{*}{$\kappa=1$} & RA1 & 39.76 \\
\cline { 2 - 3 } & RA2 & 41.33 \\
\cline { 2 - 3 } & RA3 & 36.82 \\
\hline \multirow{3}{*}{$\kappa=2$} & RA1, RA2 & 99.62 \\
\cline { 2 - 3 } & RA1, RA3 & 100.31 \\
\cline { 2 - 3 } & RA2, RA3 & 96.54 \\
\hline$\kappa=3$ & RA1, RA2, RA3 & 294.78 \\
\hline
\end{tabular}

Table III shows the computational time required for solving the problem pertaining to each number of RAs. Observe that the computational time increases dramatically along with the growth of number $\kappa$. Although the computational performance is $\kappa$-dependent, it is remarkable that the computational effort is acceptable for a hourly-based setting.

\section{CONCLUSION}

This paper proposes a bi-level model for a PDISCO to make optimal procurement with the aggregator-based DR resources in the presented real-time trading framework. The PDISCO and RAs' decisions are modeled through upper and lowerlevel problems. Combining with commercial and physical constraints, this model can explicitly address the strategies of the PDISCO's procurement about real-time market exchanging, RAs' GEP-generating, and load shedding. This proposed bilevel model is finally transformed into an MPEC for computational efficiency and tractability. The numerical results and 
impact analyses are fully discussed to validate the effectiveness of the proposed methodology.

Based on the proposed hierarchical market framework, it is relevant to note that stochastic DGs and storages could be included by aggregators. The aggregators can be classified into various types according to the characteristics of distributed energy resources. For instance, wind generations can be grouped as a type of aggregators, and batteries can be another type. Therefore, the formulations of differing aggregators vary. This topic can be studied in our future work.

\section{REFERENCES}

[1] NewYorkState, "Reforming the energy vision," NYS Department of Public Service, 2014

[2] Q. Wang, C. Zhang, Y. Ding, G. Xydis, J. Wang, and J. Østergaard, "Review of real-time electricity markets for integrating distributed energy resources and demand response," Appl. Energy, vol. 138, pp. 695-706, 2015.

[3] J. L. Mathieu, M. Kamgarpour, J. Lygeros, G. Andersson, and D. S. Callaway, "Arbitraging intraday wholesale energy market prices With aggregations of thermostatic loads," IEEE Trans. on Power Syst., vol. 30, no. 2, pp. 763-772, 2015.

[4] A. I. Negash, T. W. Haring, and D. S. Kirschen, "Allocating the cost of demand response compensation in wholesale energy markets," IEEE Trans. Power Syst., vol. 30, no. 3, pp. 1528-1535, 2015.

[5] M. Parvania, M. Fotuhi-Firuzabad, and M. Shahidehpour, "ISO's optimal strategies for scheduling the hourly demand response in day-ahead markets," IEEE Trans. Power Syst., vol. 29, no. 6, pp. 2636-2645, 2014.

[6] M. Amelin, "An evaluation of intraday trading and demand response for a predominantly hydro-wind system under nordic market rules," IEEE Trans. Power Syst., vol. 30, no. 1, pp. 3-12, 2015.

[7] B. F. Hobbs, M. C. Hu, J. G. Iñón, S. E. Stoft, and M. P. Bhavaraju, "A dynamic analysis of a demand curve-based capacity market proposal: the PJM reliability pricing model," IEEE Trans. Power Syst., vol. 22, no. 1, pp. 3-14, 2007.

[8] S. A. Gabriel, A. J. Conejo, J. D. Fuller, B. F. Hobbs, and C. Ruiz, Complementarity modeling in energy markets. New York: Springer, 2012.

[9] R. Palma-Behnke, J. L. Cerda A., L. S. Vargas, and A. Jofré, "A distribution company energy acquisition market model with integration of distributed generation and load curtailment options," IEEE Trans. Power Syst., vol. 20, no. 4, pp. 1718-1727, 2005.

[10] $\mathrm{H}$. Li, Y. Li, and Z. Li, "A multiperiod energy acquisition model for a distribution company with distributed generation and interruptible load," IEEE Trans. Power Syst., vol. 22, no. 2, pp. 588-596, 2007.

[11] J. Lopez-Lezama, A. Padilha-Feltrin, J. Contreras, and J. Munoz, "Optimal contract pricing of distributed generation in distribution networks," IEEE Trans. Power Syst., vol. 26, no. 1, pp. 128-136, Feb 2011.

[12] S. D. Manshadi and M. E. Khodayar, "A hierarchical electricity market structure for the smart grid paradigm," IEEE Trans. Smart Grid, pp. $1-10,2016$.

[13] D. T. Nguyen, H. T. Nguyen, and L. B. Le, "Dynamic Pricing Design for Demand Response Integration in Power Distribution Networks," IEEE Trans. Power Syst., pp. 1-16, 2016.

[14] A. J. Conejo, J. M. Morales, and L. Baringo, "Real-time demand response model," IEEE Trans. Smart Grid, vol. 1, no. 3, pp. 236-242, 2010.

[15] M. Zugno, J. M. Morales, P. Pinson, and H. Madsen, "A bilevel model for electricity retailers' participation in a demand response market environment," Energy Economics, vol. 36, pp. 182-197, 2013.

[16] D. Papadaskalopoulos and G. Strbac, "Decentralized participation of flexible demand in electricity markets - part I: market mechanism," IEEE Trans. Power Syst., vol. 28, no. 4, pp. 3658-3666, 2013.

[17] D. Papadaskalopoulos, G. Strbac, P. Mancarella, M. Aunedi, and V. Stanojevic, "Decentralized participation of flexible demand in electricity markets-part II: application with electric vehicles and heat pump systems," IEEE Trans. Power Syst., vol. 28, no. 4, pp. 3667-3674, 2013.

[18] M. Rahimiyan, L. Baringo, and A. J. Conejo, "Energy management of a cluster of interconnected price-responsive demands," IEEE Trans. Power Syst., vol. 29, no. 2, pp. 645-655, 2014.

[19] J. Wang, M. Shahidehpour, Z. Li, and A. Botterud, "Strategic generation capacity expansion planning with incomplete information," IEEE Trans. Power Syst., vol. 24, no. 2, pp. 1002-1010, 2009.
[20] Z. Wang, B. Chen, J. Wang, and C. Chen, "Networked microgrids for self-Healing power systems," IEEE Trans. Smart Grid, vol. 7, no. 1, pp. 310-319, 2015

[21] Z. Wang, B. Chen, J. Wang, M. M. Begovic, and C. Chen, "Coordinated energy management of networked microgrids in distribution systems," IEEE Trans. Smart Grid, vol. 6, no. 1, pp. 45-53, 2015.

[22] G. E. Asimakopoulou, A. L. Dimeas, and N. D. Hatziargyriou, "Leaderfollower strategies for energy management of multi-microgrids," IEEE Trans. Smart Grid, vol. 4, no. 4, pp. 1909-1916, 2013.

[23] C. Ruiz, A. J. Conejo, and Y. Smeers, "Equilibria in an oligopolistic electricity pool with stepwise offer curves," IEEE Trans. Power Syst., vol. 27, no. 2, pp. 752-761, 2012.

[24] S. J. Kazempour and H. Zareipour, "Equilibria in an oligopolistic market with wind power production," IEEE Trans. Power Syst., vol. 29, no. 2, pp. 686-697, 2014.

[25] R. Fernández-Blanco, J. M. Arroyo, and N. Alguacil, "Networkconstrained day-ahead auction for consumer payment minimization," IEEE Trans. Power Syst., vol. 99, no. 2, pp. 1-11, 2013.

[26] S. J. Kazempour, A. J. Conejo, and C. Ruiz, "Strategic bidding for a large consumer," IEEE Trans. Power Syst., vol. 30, no. 2, pp. 848-856, 2015.

[27] J. M. Arroyo, "Bilevel programming applied to power system vulnerability analysis under multiple contingencies," IET Gener. Transm. Distrib., vol. 4, no. 2, pp. 178-190, 2010.

[28] GAMS, [Online]. Available: http://www.gams.com/.

[29] M. E. Baran and F. F. Wu, "Network reconfiguration in distribution systems for loss reduction and load balancing," IEEE Trans. Power Del., vol. 4, no. 2, pp. 1401-1407, 1989.

[30] Nordpool, [Online]. Available: http://www.nordpoolspot.com/.

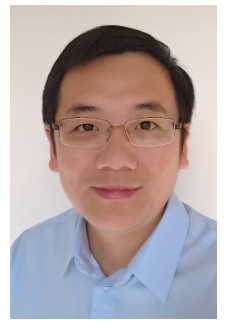

Chunyu Zhang (M'12) received the Ph.D. degree in electrical engineering from Technical University of Denmark (DTU), Denmark, in 2016. From 2006 to 2012, he joined National Power Planning Center and CLP Group as a senior engineer.

$\mathrm{He}$ is currently a postdoctoral fellow at the Department of Electric Power Engineering, Norwegian University of Science and Technology (NTNU), Norway. His research interests include power systems planning and economics, smart grid and electricity markets.

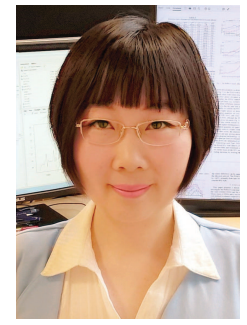

Qi Wang (M'13) received the M.S. degree from North China Electric Power University, Beijing, China, in 2008, in Electrical Control Theory and Engineering. Since then she joined East China Electric Power Design Institute as a senior engineer till 2013.

She is currently pursuing the Ph.D. degree at the Center for Electric Power and Energy, Technical University of Denmark (DTU), Denmark. Her research interests are in the fields of power systems economics, planning, reliability, electricity markets, stochastic programming, robust optimization and complementarity modeling. 


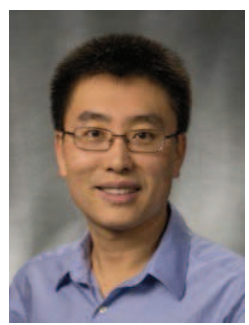

Jianhui Wang (M'07-SM'12) received the Ph.D. degree in electrical engineering from Illinois Institute of Technology, Chicago, IL, USA, in 2007. Presently, he is the Section Lead for Advanced Power Grid Modeling at the Energy Systems Division at Argonne National Laboratory, Argonne, IL, USA.

Dr. Wang is the secretary of the IEEE Power \& Energy Society (PES) Power System Operations Committee. He is an associate editor of Journal of Energy Engineering and an editorial board member of Applied Energy. He is also an affiliate professor at Auburn University and an adjunct professor at University of Notre Dame. He has held visiting positions in Europe, Australia and Hong Kong including a VELUX Visiting Professorship at the Technical University of Denmark (DTU). Dr. Wang is the Editor-in-Chief of the IEEE Transactions on Smart Grid and an IEEE PES Distinguished Lecturer. He is also the recipient of the IEEE PES Power System Operation Committee Prize Paper Award in 2015.

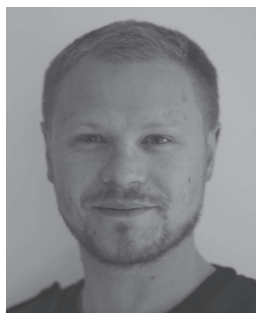

Pierre Pinson (M'11-SM'13) received the M.Sc. degree in applied mathematics from the National Institute for Applied Sciences (INSA Toulouse, France) and the Ph.D. degree in energetics from Ecole des Mines de Paris (France). He is a Professor at the Technical University of Denmark, Centre for Electric Power and Energy, Department of Electrical Engineering, also heading a group focusing on Energy Analytics \& Markets. His research interests include among others forecasting, uncertainty estimation, optimization under uncertainty, decision sciences, and renewable energies.

Prof. Pinson acts as an Editor for the International Journal of Forecasting, and for Wind Energy.

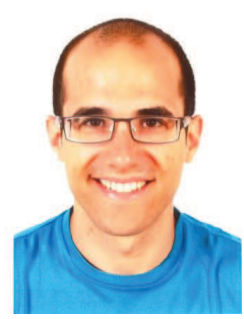

Juan M. Morales (M'11-SM'16) received the Ingeniero Industrial degree from the Universidad de Málaga, Málaga, Spain, in 2006, and a Ph.D. degree in Electrical Engineering from the Universidad de Castilla-La Mancha, Ciudad Real, Spain, in 2010. $\mathrm{He}$ is currently an associate professor in the Department of Applied Mathematics and Computer Science at the Technical University of Denmark, Kgs. Lyngby, Denmark.

His research interests are in the fields of power systems economics, operations and planning; energy analytics and smart grids; decision-making under uncertainty, and electricity markets.

Jacob Østergaard (M'95-SM'09) received the M.Sc. degree in electrical engineering from the Technical University of Denmark (DTU), Lyngby, Denmark (DTU), in 1995. He is a Professor and Head of the Centre for Electric Power and Energy, Department of Electrical Engineering at DTU. From 1995 to 2005, he worked for the Research Institute for the Danish Electric Utilities.

Prof. Østergaard is serving in several professional organizations including the EU SmartGrids advisory council. His research interests include system integration of wind power, control architecture for future power systems, and demand side. 